\title{
SPLITTING OF PERIODIC COMETS
}

\author{
GIUSEPPE FORTI \\ Osservatorio Astrofisico di Arcetri, 50125 Firenze, Italy
}

\begin{abstract}
Among the comets that were observed to break in two or more fragments, only a few of them are periodic. So far the dynamic study of the relative motion of a secondary nucleus with respect to the primary has supposed that a cometary fragment is subject to a small and continuous radial nongravitational force after separation at rest. This force acts against the solar attraction and varies according to an inverse square law. A small impulse at break up may also be invoked in some case. A different approach is followed in this paper when dealing with a fragment of a periodic comet: after separation the motion of a secondary nucleus is characterized by nongravitational forces which vary according to the same $g(r)$ law currently used for the primary.

Results of the study of comets P/Biela and P/du Toit- Hartley show that the motion of their fragments after separation is characterized by nongravitational parameters which are larger than those of the parent bodies. Both fragments lasted for about 2 full revolutions and three returns.
\end{abstract}

Key words: Comets - Splitting - Nongravitational Forces

\section{Introduction}

A great deal of research and work on split comets has been done by Sekanina, who introduced a new idea on the relative motion of split nuclei. According to Sekanina [5] the motion of a secondary nucleus that divided from the parent body is dominated not by an impulse at separation, but by a small and continuous differential radial nongravitational force relative to the main body after separation at rest. The introduction of this small force acting on comet fragments and the time of splitting as the only parameters gave in a very simple dynamical model yielding better results in the representation of the separations of the secondary nuclei of split comets. However Sekanina [6] found that in some cases there were second order effects and this led to two possibilities: a) the existence of a small impulse at break up; b) deviations of the deceleration vector from the radial direction and/or changes of its magnitude from the adopted law. He opted for the first possibility in his paper.

\section{Results from a new approach}

An attempt to study with Sekanina's model the motion of the companion of periodic comet $d u$ Toit - Hartley ( for which photographic observations were available, Forti [1] ) led to relatively high O-C residuals. The assumption that $P / d u$ Toit Hartley companion was moving on an orbit similar to that of the main comet, but with different perihelion time, led instead to a decrease of the residuals. A decent fit to the observations was found by assuming that the secondary nucleus moved on an orbit identical to that of the primary but with perihelion time increased by an empirical $\Delta t$ of +0.367 days. Such $\Delta t$ in the perihelion time of the secondary nucleus might be explained by the action of nongravitational forces during one or more perihelion passages since we are dealing with a short period comet. A fictitious break up time was found before perihelion in 1982 since most of the previous effects of nongravitational forces were included in $\Delta t$. The computed differential radial acceleration was relatively high. 


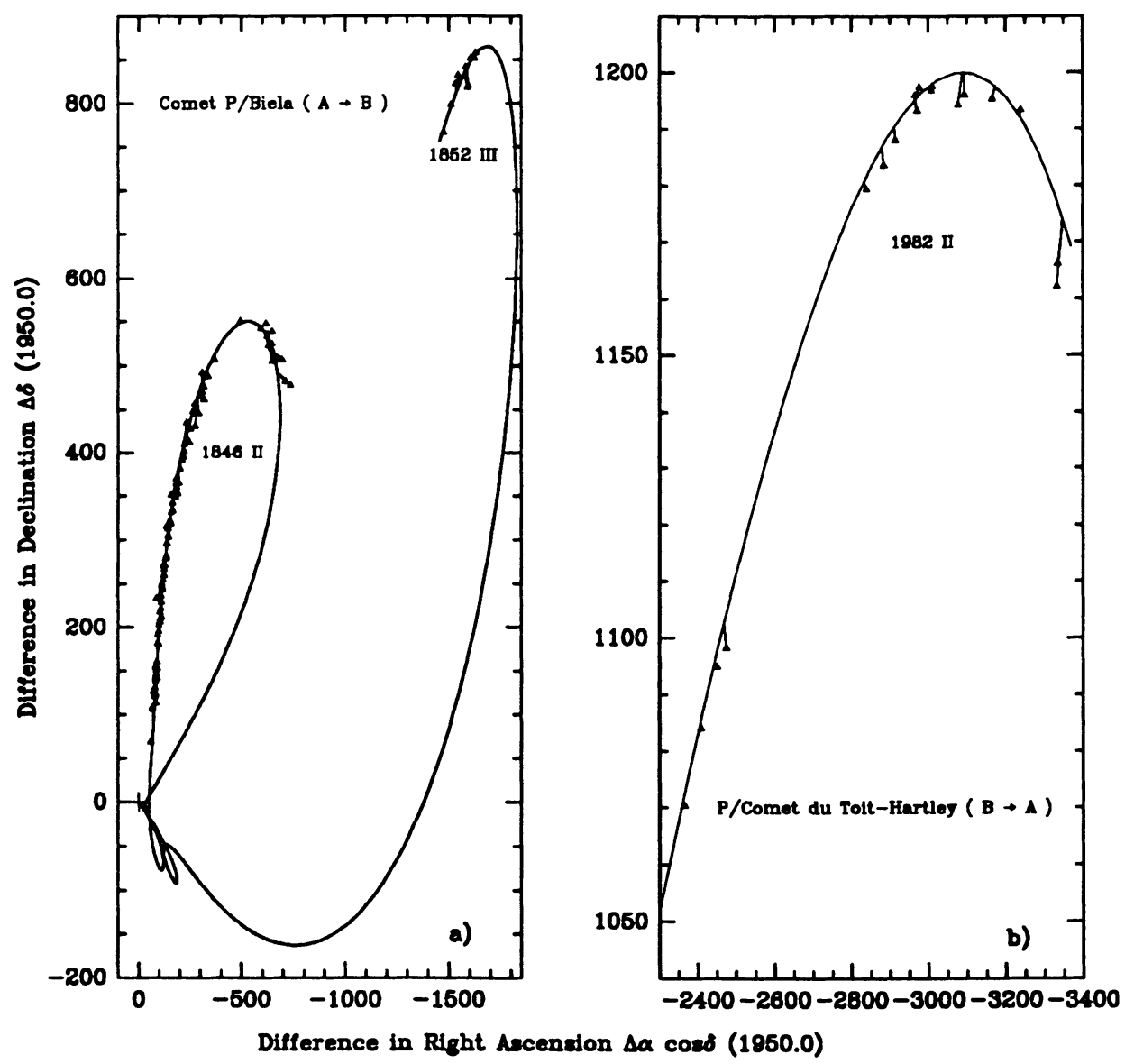

Fig. 1. Observed (solid triangles), computed differences (solid lines) in Right Ascension and Declination for comets $P /$ Biela and $P / d u$ Toit - Hartley.

This interpretation of $\Delta t$ led me to suppose that the radial model was better suited for long period comets and that for short period comets it was necessary to consider the full effect of nongravitational forces, acting only for a limited period of time near each perihelion.

A program was written to find the time $T$, of break up and the nongravitational constants of a secondary nucleus according to the so called "Style II" or standard model ( Marsden et al., [4] ) supposing to know the nongravitational motion of the primary.

This program was first run on the observations of $P$ / Biela 's fragment ( a comet seen at two perihelion passages with a companion ) with eminent success since it was possible to link the separation data of the secondary nucleus in 1846 and 1852 . 
Table 1 summarizes the results for all the studied comets. Left to right: comet name; splitting time $T_{0}$; difference $T_{2}-T_{0}$ in days; period; perihelion distance; heliocentric distance at break up; nongravitational force $F_{i}\left(=A_{i} g(r)\right)$ constants $A_{i}$ for the main ( A ) and the secondary ( B ) nucleus ; nucleus identification; mean residual; number of observations; periods of observations; and minimum and maximum separation.

According to the present study the splitting of $P /$ Biela occurred about a month after the unseen perihelion passage in $1839\left(T_{0}=1839 / 07 / 23.32\right)$. The error in time $T$, is smaller than the integration step of 1 day. The identification of the fragments confirms that adopted by Marsden ( Marsden and Sekanina, [3] ), the case $A \rightarrow B$ is listed in Table 1 and only for completeness $I$ provide the results for the other alternative $(B \rightarrow A)$. These last results however should be dismissed for it is difficult to believe that after break up the two nuclei rotate in opposite directions as deduced from the sign of $A_{2}$. In Fig. 1a I plotted observed separations and computed differences for this comet.

Successively periodic comet $d u$ Toit - Hartley was studied. For this comet the results are conclusive, but more indeterminate than those found for $P$ /Biela because the split nucleus was seen only during the 1982 passage. The determination of time $T_{\text {, }}$ is rather poor for this comet, depending somewhat on the choice of the initial nongravitational parameters. However this procedure sets un upper limit for a break up date around Nov. 4, 1970, well before the unseen perihelion passages of this comet in 1971 and in 1977. The quantities shown for this comet on Table 1 are those found for a particular couple of initial nongravitational parameters. In this case the $T$, found is the upper limit from this particular run. In Fig. 1b I plotted observed and computed separations for this comet.

Another periodic split comet for which there are measured separations is $P /$ Taylor. For this comet there are so far no published nongravitational parameters and I tried to find them taking the observations from 1976 up to the last published photographic data since the recovery of this comet in 1990. The computed nongravitational parameters seem to be plausible because with them it is possible to match the perihelion time in 1916 ( Marsden, [2] ). However they were computed solely on all the post-perihelion data.

The results obtained for this comet using the new model show a break up time $T$, on $1915 / 10 / 26$ with an error of \pm 2 days. The nongravitational parameters instead seem to be rather uncertain, suggesting that there might be some intrinsic difficulty in dealing with this comet ( Marsden, [2], p. 53 ).

\section{Conclusions}

Abandoning the model of a continuous differential radial nongravitational force (force that, by definition, varies like $1 / r^{2}$ ), I considered a fragment of a periodic comet, immediately after division from the parent body, to be a new periodic comet and its motion characterized by nongravitational forces like that of the main comet.

The nongravitational motion of the secondary nucleus of three periodic comets was successfully computed. The nongravitational parameters found for these new comets are larger than those of the parent body as we should expect, for at each 
TABLE I

\begin{tabular}{|c|c|c|c|c|}
\hline 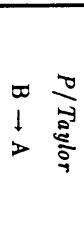 & 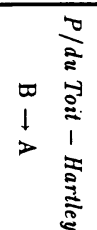 & $\begin{array}{ll}\infty & 0 \\
1 & 0 \\
> & \frac{0}{2}\end{array}$ & 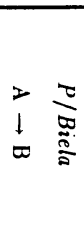 & 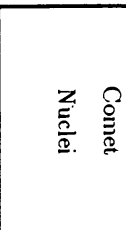 \\
\hline$\frac{\overrightarrow{0}}{\frac{0}{0}}$ & के & 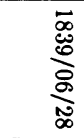 & 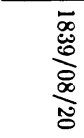 & 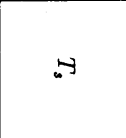 \\
\hline $\begin{array}{l}1 \\
\stackrel{1}{1} \\
\text { के }\end{array}$ & 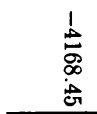 & 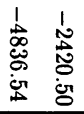 & 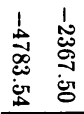 & 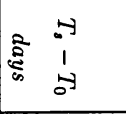 \\
\hline is & $\underset{i v}{\mathrm{~N}}$ & as & $\dot{\sigma}$ & 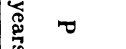 \\
\hline غூ & $\overrightarrow{\tilde{N}}$ & $\begin{array}{l}0 \\
\infty \\
\infty\end{array}$ & $\stackrel{0}{\infty}_{\infty}$ & $\vec{a} e$ \\
\hline$\vec{\infty}$ & $\stackrel{\omega}{\dot{\omega}}$ & : & : & 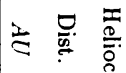 \\
\hline 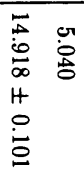 & $\begin{array}{ll}\overrightarrow{\dot{\omega}} & 0 \\
\text { o } & \text { 히 } \\
\text { H } & 0 \\
0 & \\
\dot{8} & \end{array}$ & 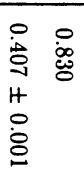 & 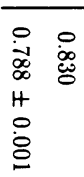 & 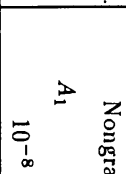 \\
\hline 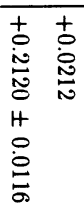 & 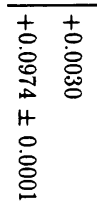 & 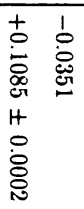 & 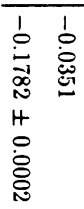 & 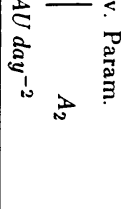 \\
\hline$\omega>$ & $\varpi>$ & $\infty>$ & $\varpi>$ & 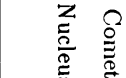 \\
\hline 5 & $\because$ & $\bar{i}$ & हे & 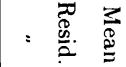 \\
\hline$=$ & $\overrightarrow{0}$ & $\overline{0} \equiv$ & ๘ & 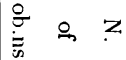 \\
\hline 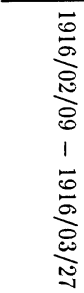 & 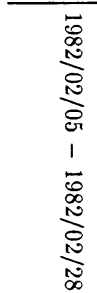 & 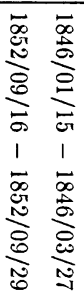 & 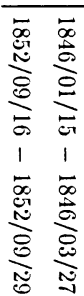 & 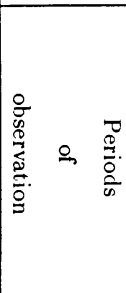 \\
\hline $\begin{array}{l}\overrightarrow{1} \\
\vec{y}\end{array}$ & 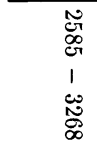 & 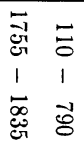 & $\begin{array}{ll}\vec{j} & \Xi \\
\mathrm{w} & \Xi \\
1 & 1 \\
\vec{\infty} & \ddot{2}\end{array}$ & 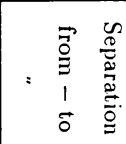 \\
\hline
\end{tabular}


return the secondary nucleus shows a $\Delta t$ correction larger than that of the primary. The cases of the fragments of comets $P /$ Biela and $P / d u$ Toit - Hartley seem to be very similar since they lasted for about 2 full revolutions and three returns. The former comet fragment was seen at two successive returns after break up, the latter only at the last one. For $P$ / Taylor the results are rather uncertain like the observed separation data and the nongravitational constants for the main comet. Since in this case the nongravitational force seems to be essentially radial, even Sekanina's model works equally well.

A comparison with earlier results ( Sekanina [6] ) shows that for comets $P /$ Biela and $P /$ Taylor the splitting time is rather different: for both comets he found a later $T_{s}$, respectively 1840 May 25 and 1915 December 8 ( see Table 1 ). The radial accelerations are different, but not as much as we would expect from two different models. This is especially true for $P /$ Taylor for which he computes a radial acceleration of about 34 units against the actual 50 units ( 1 unit $=2.96$ $10^{-9} A U d a y^{-2}$ ).

\section{References}

[1] Forti, G. (1989), The motion of several periodic comets., Astron. Astroph., 215, 381-386.

[2] Marsden, B. G. (1986), Catalogue of Cometary Orbits 5th ed., IAU Central Bureau for Astron. Telegrams, Cambridge, Mass., U.S.A.

[3] Marsden, B. G. and Sekanina, Z. (1971), Comets and nongravitational forces. IV., Astron. J.,76,1135-1151.

[4] Marsden, B. G., Sekanina, Z. and Yeomans, D. K. (1973), Comets and nongravitational forces. V, Astron. J.,78, 211-225.

[5] Sekanina, Z. (1977), Relative motions of fragments of the split comets. I. A new approach, Icarus, 30, 574-594.

[6] Sekanina, Z. (1982), The problem of split comets in review. In Comets, L. L. Wilkening, Ed. , 251-287. Univ. of Arizona Press, Tucson. 